\title{
Asymptotic series for the scattering operator and asymptotic unitarity of the space \\ cut-off interactions
}

by

Sergio Albeverio

and

Raphael Høegh-Krohn

\author{
Institute of Mathematics \\ University of Oslo \\ Blindern, Oslo 3, Norway
}

A B S T R A C T

We construct the asymptotic expansions in powers of the coupling constant $\lambda$ for the asymptotic fields and the scattering operator $S$ for self-coupled Boson fields with space cut-off polynomial interaction in two space-time dimensions. These asymptotic expansions are then used to prove that $S^{*} S=S^{*}=11$ in the sense of asymptotic power series in $\lambda$, on a dense set of states.

The results apply also, under the additional assumption of an ultraviolet cut-off, to large classes of boson-boson, fermi-boson and fermi-fermi interactions as well as to boson nonpolynomial interactions (in all space-time dimensions). 
1. Introduction.

Whereas the existence of the basic quantities for a mathematical description of the scattering of particles has been proven for a number of interactions, both for systems of finitely many and for systems of infinitely many particles 1), the problem of the unitarity of the scattering operator has been tackled successfully only for certain quantum mechanical systems of finitely many particles and very restricted forces 2) and, as far as field theoretical models are concerned, for the case where no pure creation terms are present in the interaction i.e. for models without vacuum polarization [6]. 3) In this paper we study the S-matrix for the space cut-off polynomial boson interactions in two spacetime dimensions [7]. These $P(\varphi)_{2}$ interactions have been studied intensively in recent years, especially by J. Glimm and A. Jaffe, and shown to have limits, when the space cut-off is taken away, which satisfy all the Wightman axioms for a local, relativistic covariant theory of quantized fields.

In [8] one of us has constructed the asymptotic fields for these models, for the case of a space cut-off interaction. 4)

In this paper we prove that these asymptotic fields are equal, in the sense of asymptotic series, to a power series in the coupling constant $\lambda$, on a dense domain of the lock space. 5)

The scattering operator $S$ is defined in terms of the asymptotic fields. Using the asymptotic series for the asymptotic fields we then prove that $S$ is asymptotic for $\lambda=0$ to an asymptotic series in powers of $\lambda$, on a dense domain. This yields then asymptotic series for all S-matrix elements between dense sets

1) See e.g. [1],[2] and the references given therein.

2) See e.g. [2], [3] and [4], [5], and the references quoted therein.

3) References for models somewhat inbetween the two mentioned classes, like e.g. external field models and lee-type models are mentioned e.g. in [16].

4) Related results for the special case $P(\varphi)=\varphi^{4}$ have been obtained also in [9].

5) Such asymptotic expansions have been derived in [10] for space and ultraviolet cut-off relativistic fermions interactions. The case of Nelson's type models is treated in [1b] and the case of non polynomial boson models in [11b]. 
of states. In particular it follows that the S-matrix is not trivial. The asymptotic series for $S$ are then used, in section4, to study the operators $S * S$ and $S S^{*}$ on a dense set of vectors. We prove that $S^{*} S$ and $S^{*}$ are both asymptotic, for small values of $\lambda$, to the identity operator, on the chosen dense set of states. This then proves the unitarity of the S-matrix in the sense of asymptotic series. The results use essentially a strong control on the Hamiltonian, such as the one provided by Rosen's higher order estimates [12], and the existence of the asymptotic fields [8]. The same information is available for a large class of space and ultraviolet cut-off interactions, in any space timedimensions. Our results extend therefore to such interactions, including the bose-bose, bose-fermi and fermi-fermi polynomial interactions of [10], the NeIson's type interactions of [1] and the non polynomial interactions of [11].

2. The models, the asymptotic fields, the wave operators and the scattering operator.

Let $\mathscr{F}$ be the Fock space for scalar bosons in two spacetime dimensions, with mass $m>0$. $\mathscr{F}$ is the direct sum $\mathscr{F}=\mathscr{F}^{\infty} \mathscr{F}^{n}$, where $\mathcal{F}^{n}$ is the space of all symmetric square integrable functions of $\mathrm{n}$ (momentum) variables. The time zero boson field $\varphi(x)$ is given in terms of annihilationcreation operators on $\mathscr{F}$ by

$$
\varphi(x)=(4 \pi)^{-\frac{1}{2}} \int e^{i k x}[a *(k)+a(-k)] \mu(k)^{-\frac{1}{2}} d k,
$$

where $\mu(k)=\sqrt{k^{2}+m^{2}}$ and $x, k$ run over the one dimensional space $\mathbb{R}$. The annihilation-creation operators satisfy

$$
\left[a(k), a^{*}\left(k^{8}\right)\right]=\delta\left(k-k^{1}\right) \text {. }
$$


Let $\mathrm{H}_{\mathrm{O}}$ be the free Hamiltonian in $\mathscr{F}$ and

$$
\lambda V=\lambda \int_{\mathbb{R}} g(x): P(\varphi(x)): d x
$$

be the interaction, where $\lambda$ is the (real) coupling constant, $g(x)$ is a smooth non negative function of compact support, $P(\alpha)$ is a polynomial bounded from below, and $: P(\varphi(x))$ : is the correspondent Wick-ordered polynomial in the field (see e.g. [7]).

It is proven (see e.g. [7]) that $H=H_{0}+\lambda V$ is essentially selfadjoint on the intersection $D\left(H_{O}\right) \cap D(V)$ of the domains of the self-adjoint operator $H_{O}$ and the symmetric operator $V$. Moreover the following power estimates have been proven by I. Rosen [12]:

$$
\begin{gathered}
H_{0}^{2} \leq a(H+b)^{2}, \quad \underline{\mathbb{N}}^{j} \leq a_{j}\left(H+b_{j}\right)^{j}, \\
\left\|(\underline{N}+1)^{-\alpha} V(\underline{N}+1)^{-\beta}\right\|<\infty,
\end{gathered}
$$

the latter for the case $\alpha+\beta \geq p, 2 p$ being the degree of the polynomial $P(\alpha)$ which gives the interaction. $\mathbb{N}$ is the particle number operator, $a, b, a_{j}, b_{j}$ are constants, $j=1,2, \ldots$. For $h$ in $\mathscr{F}^{-1}$ the annihilation-creation operators $a^{\frac{\pi}{11}}(h)$, where $a^{*}$ stands for a or $a^{*}$, are well defined closed operators on a domain containing the domain $D\left(\underline{\mathbb{N}}^{\frac{1}{2}}\right)$. They are related to the $a^{*}(k)$ by the usual formal relations

$$
a^{\frac{\pi}{\pi}}(h)=\int a^{\#}(k) h(k) d k \text {. }
$$

The $a(\bar{h}), a^{*}(h)$ are mutually adjoints and satisfy the commutation relations

$$
\left[a(\bar{h}), a^{*}(g)\right]=(h, g),
$$

on a domain containing $D(\underline{\mathbb{N}})$.

In Ref. [8] the second named author proved the following: 


\section{Theorem 2.1}

For any $h$ in $\mathscr{F}^{1}$ and any time $t \in \mathbb{R}$, the operators

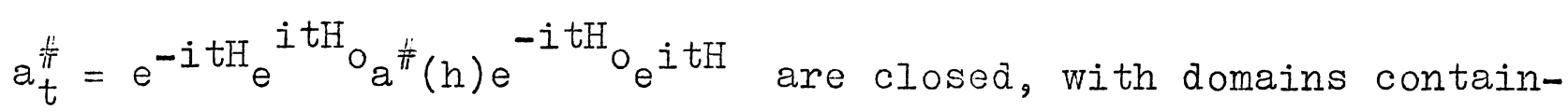
ing the domain $\mathrm{D}\left(\mathrm{H}+\mathrm{b}_{1}\right)^{\frac{1}{2}}$ of $\left(\mathrm{H}+\mathrm{b}_{1}\right)^{\frac{1}{2}}$, and converge strongly as $t \rightarrow \pm \infty$ on $D\left(H+b_{1}\right)^{\frac{1}{2}}$. Call $a_{ \pm}^{\frac{4}{\#}}(h)$ these strong limits. They satisfy the same commutation relations on the domain of $H$ as do $a^{\frac{H}{11}}(h)$ on the domain of $\mathrm{H}_{\mathrm{O}}$, i.e.

$$
\left[a_{ \pm}(\bar{h}), a_{ \pm}^{*}(g)\right]=(h, g) \text {. }
$$

Moreover $H$ and $a_{ \pm}^{\#}(g)$ satisfy the same commutation relations as do $H_{0}$ and $a^{\#}(h)$, in the sense that

$$
e^{i t H} a_{ \pm}^{\#}(h) e^{-i t H}=a_{ \pm}^{\#}\left(h_{ \pm t}\right)
$$

where $h_{t}(k)=e^{i t \mu(k)} h(k)$, and + goes with $a^{*}$, - with a. Let $\Omega$ be the eigenvector to the simple, isolated, lowest eigenvalue $\mathrm{E}$ of $\mathrm{H}, 6$ )

Let $\mathscr{F}_{ \pm}$be the subspaces generated by applying all polynomials in $a_{ \pm}^{*}(\bar{h})$ to $\Omega$. Then $\mathcal{F}$ can be decomposed as a tensor product $\mathscr{F}=\mathscr{F}_{ \pm} \otimes V_{ \pm}$, where $\Omega \otimes V_{ \pm}$is the closed subspace of $\mathscr{F}$ annihilated by $a_{ \pm}(h)$ for all $\bar{h} \in \mathscr{F}^{1}$. Relative to this tensor decomposition the operator $\mathrm{H}-\mathrm{E}$ has the form $\mathrm{H}-\mathrm{E}=\mathrm{H}_{0}^{ \pm} \otimes \Pi+\pi \otimes \mathrm{H}_{ \pm}^{\circ}$, where $\mathrm{H}_{0}^{ \pm}$is the free energy operator in $\mathscr{F}_{ \pm}$and $\pi \otimes \mathrm{H}_{ \pm}^{\mathrm{O}}$ is the restriction of $(H-E)$ to the invariant subspace $\Omega \otimes V_{ \pm}$, which is positive, with finitely dimensional spectral decomposition 7$)$ on the interval $[0, m-\epsilon]$, for any $\epsilon>0$.

6) For the proof that the bottom of the spectrum of $H$ is a simple, isolated eigenvalue, see e.g. [7].

7) See e.g. [7]. 
For any operator A, given in terms of the annihilation-creation operators $a^{\#}(p)$, we set

$$
A_{t}=e^{-i t H} e^{i t H_{0}} e^{-i t H_{0}} e^{i t H}
$$

Let now $A$ be of either of the forms

$$
\begin{aligned}
& A=\left[V\left(t_{j}\right),\left[V\left(t_{j-1}\right), \ldots,\left[V\left(t_{1}\right), B\right] \ldots\right]\right] \text { or } \\
& A=\alpha^{O}\left(t_{j}\right)\left(\left[V\left(t_{j}\right), \alpha^{O}\left(t_{j-1}\right)\left(\left[V\left(t_{j-1}\right), \ldots, \alpha^{O}\left(t_{1}\right)\left(\left[V\left(t_{1}\right), B\right]\right) \ldots . .0\right]\right)\right]\right),
\end{aligned}
$$

where $\alpha^{o}(t)(c)=e^{i t H_{O}} e^{-i t H_{O}}$, for any operator $C$, and $V(t)=$ $=\alpha^{\circ}(-t)(V)=e^{-i t H_{0}} V^{i t H_{O}}$, and $B=a^{*}\left(h_{1}\right) \ldots a^{*}\left(h_{k}\right)$, with $\mathrm{h}_{i} \in \mathscr{F}^{1}, i=1, \ldots, \mathrm{k}$. We malie also the convention to allow for the value $j=0$ in (2.7), setting $A=B$ in this case. For $j=1,2, \ldots$, call $D_{j / 2}$ the domains of the operatoos $\left(H+b_{j}\right)^{j / 2}$.

Let again $2 p$ be the degree of the lower bounded polynomial $P(\alpha)$ which gives the interaction. For any $\Psi \in D_{j p+\frac{k}{2}}$ the following estimates are a simple consequence of the higher order estimates (2.4) and the fact that the $a_{t}^{\#}\left(h_{i}\right)$ are closed operators with domain containing $D_{\frac{1}{2}}$ :

$$
\left\|A_{t} \Psi\right\| \leq C(j, k)\left\|h_{1}\right\| \ldots\left\|h_{k}\right\|\left\|\left(H+b_{2} j p+k\right)^{j p+\frac{k}{2}} \psi\right\|,
$$

where $C(j, k)$ is independent of $t, \Psi_{,} t_{1}, \ldots, t_{j}, h_{i}, i=1 \ldots k$. These estimates are thus, in particular, uniform in the time variables $t_{,} t_{1}, \ldots, t_{j}$.

\section{Iemma 2.1}

Let $F$ be any bounded operator on $\mathscr{F}$ and $\mathrm{F}_{t}=e^{-i t H_{1}} e^{i t H} O_{\mathrm{Fe}} \mathrm{itH}_{\mathrm{O}} \mathrm{e}^{\mathrm{itH}}$. Then $\mathrm{F}_{t}$ converges strongly as 
$t \rightarrow \pm \infty$ to bounded operators $\mathrm{F}_{ \pm}$.

Proof: By Theorem 2.1, $a_{t}^{*}(h)+a_{t}(\bar{h})$ converges strongly as $t \rightarrow \pm \infty$, hence by the Trotter convergence theorem $e^{i\left(a_{t}^{*}(h)+a_{t}(\bar{h})\right)}$ converges strongly. Therefore for any continuous function $F$ of the time zero field $\varphi$ we have that $F_{t}$ converges strongly. But these operators are strongly dense in the space of all bounded operators. Having the strong convergence for a strongly dense set, we get strong convergence for all bounded operators using the uniform boundedness of the mapping $F \rightarrow F_{t}$ (since $\left\|F_{t}\right\|=$ $=\|F\|)$.

\section{Lemma 2.2}

For any $\Psi \in \underset{p(j+1)+\sum^{D}}{\text { and }} h_{i} \in \mathscr{F}^{-1} \cap C^{\infty}\left(H_{0}\right)$, one has $A_{t} \Psi=A \Psi-i \lambda \int_{0}^{t} e^{-i s H}\left[V, e^{i s H_{0}} \mathrm{Ae}^{-i s H_{0}}\right] e^{i s H_{\Psi} d s}$, where the integral is a strong one.

Proof: The proof is completely similar to the one of Lemma 2 in Ref.[8] and uses (2.8) together with the essential self-adjointness of $H$ on $D\left(H_{O}\right) \cap D(V)$.

(2)

Proceding now as in the proof of Theorem 2.1, given in Theorem 1 of Ref.[8], we first prove the following:

\section{Lemma 2.3}

Let $A$ be as in (2.7). The following estimate holds in the case where all the functions $h_{1}, \ldots, h_{k}$ belong to the dense subspace of $\mathscr{F}^{1}$ consisting of $C_{0}^{\infty}(\mathbb{R})$ functions which vanish in a neighborhood of the origin: 
$\|\left[V, e^{i t H} O_{A e} e^{-i t H} O \Psi \psi=\left\|[V(t), A] e^{-i t H} o_{\Psi}\right\| \leq C(j, k, \mathbb{M}, \Psi)(1+|t|)^{-\mathbb{M}}\right.$, for any $\Psi \in \underset{p(j+1)+\frac{k}{2}}{D}$, any integer $\mathbb{N}$, where $C(j, k, \mathbb{M}, \Psi)$ is independent of $t, t_{1}, \ldots, t_{j}$.

Proof: One has $\left[\mathrm{V}, e^{i t H} o_{A e} e^{-i t H} 0\right] \Psi=e^{i t H} 0[V(t), A] e^{-i t H} o_{\psi} . A$ is given by the multiple commutator $(2.7)$ and expanding these commutators and Wick ordering after expansion every term we get A as a sum of Wick ordered monomials $P_{i} \cdot{ }^{8)}$ The commutator of $V(t)$ with any term $P_{i}$ is itself a sum of terms. After Wick ordering of the terms there remains, since $V$ is spatially cut-off, terms of the form:

$$
\begin{aligned}
T_{i}=\int f\left(p, p_{1}, \ldots, p_{r}\right) e^{i t \mu(p)} a^{*}\left(p_{1}\right) \ldots a *\left(p_{S}\right) \\
a\left(p_{S+1}\right) \ldots a\left(p_{r}\right) d p d p_{1} \ldots d p_{r},
\end{aligned}
$$

where $f$ is a $c^{\infty}$ square integrable function (which may depend also on some of the variables $t_{1}, \ldots, t_{j}$ and on the $h_{1}, \ldots, h_{k}$ ) of compact support in $\mathrm{p}$ and vanishing in a neighborhood of $p=0$. Such a term is estimated in the usual way (see e.g. [8], $[9],[10])$ by

$$
\left\|\mathrm{T}_{i}(\mathrm{~N}+1)^{-(\mathrm{s}+\mathrm{r}) \frac{1}{2}}\right\| \leq \mathrm{C}_{\mathbb{M}}(1+\mid \mathrm{t} !)^{-\mathbb{M}},
$$

where $C_{\mathbb{M}}$ is independent of $t$.

Since $\left[\mathrm{V}, \mathrm{e}^{i t \mathrm{H}_{\mathrm{A}}} \mathrm{e}^{-i t \mathrm{H}_{\mathrm{O}}}\right]$ is, by above argument, a sum of finitely many terms of the form $\mathbb{T}_{i}$, the Lemma is proven.

\section{Lemma 2.4}

For any $h_{i} \in \mathcal{F}^{1}, i=1, \ldots, k$, the strong limits as $t \rightarrow \pm \infty$ of the operator $A_{t}$ defined by $(2.6),(2.7)$ exists on the domain

8) For definitions, see e.g. [7], [13]. 


$$
\begin{aligned}
& D_{p j+\frac{k}{2}} \text { Calling } A_{ \pm} \text {these limits we have, for any } \Psi \in D_{p j+\frac{k}{2}} \\
& A_{ \pm} \Psi=\underset{t \rightarrow \pm \infty}{s-l i m} A_{t} \Psi=A \Psi-i \lambda \int_{0}^{ \pm \infty} e^{-i s H}\left[V, e^{i s H_{0}} A e^{-i s H_{0}}\right] e^{i s H_{\Psi}} d s
\end{aligned}
$$

where the integral is strongly convergent.

Moreover the following equality holds:

$$
e^{-i s H_{ \pm}} e^{i s H_{\Psi}}=\left(e^{-i s H_{0}} A e^{i s H}\right)_{ \pm}^{\Psi} \text {. }
$$

Corollary: Call $\mathscr{A}$ the polynomial algebra generated by the identity operator and all possible monomials $a^{\# \prime}\left(h_{1}\right) \ldots a^{\#}\left(h_{k}\right)$, with arbitrary $h_{i} \in \mathscr{F}^{1}, i=1, \ldots, k$ and arbitrary $k$. For any

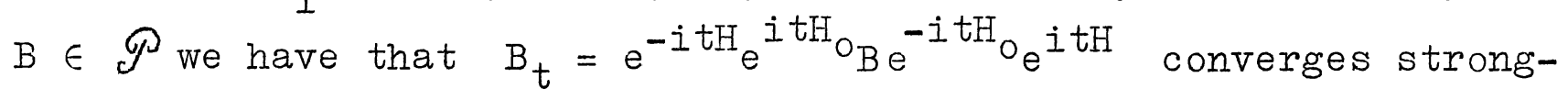
Iy as $t \rightarrow \pm \infty$ on a dense domain, $D_{k / 2}$, where $k$ is the degree of the polynomial B.

Proof: The lemma and its Corollary are immediate consequences of Lemma 2.2 and Iemma 2.3, in the case where all the $h_{i}$ belong to the dense set of $\mathscr{F}^{1}$ described in Iemma 2.3. The extension of this convergence to the case of general $h_{i}$ in $\mathscr{F}^{1}$ is a consequence of the uniform bounds (2.8). The last equality is proven by observing that, on one hand, s-lim $e^{-i s H_{A}} e_{t} e^{i s H_{\Psi}}=$ $=e^{-i s H_{ \pm}} e^{i s H_{\Psi}}$, on the other hand $e^{-i s H_{A}} e^{i s H_{\Psi}}=$ $=\left(e^{-i s H_{0}}{ }^{i s H}{ }\right)_{S+\tau}{ }^{\Psi}$, hence the strong limit is also equal $\left(e^{-i s H_{0}} \mathrm{Ae}^{i s H_{0}}\right)_{ \pm}^{\Psi}$.

Define now the wave operators $W_{ \pm}$as the isometries which are the unique extensions of the operators defined on $a^{+}\left(h_{1}\right) \ldots$ . $a *\left(h_{k}\right) \Omega_{0} \quad$ by

$$
W_{ \pm} a^{*}\left(h_{1}\right) \ldots a^{*}\left(h_{k}\right) \Omega_{0}=a_{ \pm}^{*}\left(h_{1}\right) \ldots a_{ \pm}^{*}\left(h_{k}\right) \Omega,
$$


where $\Omega_{0}$ is the Fock vacuum, $\Omega$ is the lowest eigenvalue of $H$ and $h_{i}$ are arbitrary in $\mathscr{F}^{1}, i=1, \ldots, k, k=1,2, \ldots$. The wave operators $W_{ \pm}$have therefore domain $\mathcal{F}$ and range $\mathcal{F}_{ \pm}$.

Lemma 2.5

Let $A$ any operator of the form (2.7). Then

$$
W_{ \pm} A \Omega_{0}=A_{ \pm} \Omega
$$

where $A_{ \pm}$are the strong limits of $A$ as $t \rightarrow \pm \infty$, given by Lemma $2 . \frac{ \pm}{4}$.

Proof: We first prove the lemma for the case where $A$ is of the form $A=a^{i t}\left(h_{1} \ldots a^{\#}\left(h_{k}\right)\right.$, with $h_{i} \in \mathscr{F}^{1}, i=1 \ldots k$. One has then $A_{t}=a_{t}^{\frac{\pi}{\pi}}\left(h_{1}\right) \ldots a_{t}^{\#}\left(h_{k}\right)$ and, by Lemma 2.4: $A_{t} \Omega \rightarrow A_{ \pm} \Omega$ as $t \rightarrow \pm \infty ;$ similarly, with $A^{\prime}=a^{\frac{\|}{\|}}\left(h_{2}\right) \ldots a^{\frac{\| \prime}{f t}}\left(h_{k}\right)$ one has $A_{t} \Omega \rightarrow A_{ \pm}^{\prime} \Omega$, where all convergencesare strong. On the other hand, by Theorem 2.1 for any $\Phi \in D_{\frac{1}{2}}$

$$
\left(\Phi, a_{t}^{*}\left(h_{1}\right) \ldots a_{t}^{*}\left(h_{l}\right) \Omega\right)=\left(a_{t}\left(\bar{h}_{1}\right) \Phi, A_{t}^{i} \Omega\right)
$$

converges, as $t \rightarrow \pm \infty$, to

$$
\left(a_{ \pm}\left(\bar{h}_{1}\right) \Phi, A_{ \pm} \Omega\right)
$$

This proves $a_{ \pm}\left(\bar{h}_{1}\right) * A_{ \pm}^{i} \Omega=A_{ \pm} \Omega$.

Iteration of the same argument yields then

$$
a_{ \pm}\left(\bar{h}_{1}\right)^{*} \ldots a_{ \pm}\left(\bar{h}_{k}\right) * \Omega=A_{ \pm} \Omega
$$

on the other hand, since the $a_{ \pm}^{\#}$ act on $\mathscr{F}_{ \pm} \cap D_{k / 2}$ in the same way as free annihilation-creation operators, one has

$$
a_{ \pm}\left(\bar{h}_{1}\right)^{*} \ldots a_{ \pm}\left(\bar{h}_{k}\right) * \Omega=a_{ \pm}^{*}\left(h_{1}\right) \ldots a_{ \pm}^{*}\left(h_{k}\right) \Omega
$$

and this, together with $(2.10)$ and the definition of $W_{ \pm}$, yields the equality of the Iemma, for such $A$. 
On the other hand any operator of the form (2.7) is a sum of operators of the form

$$
A_{i}=\int f\left(p_{1}, \ldots, p_{r}\right) a *\left(p_{1}\right) \ldots a *\left(p_{S}\right) a\left(p_{S+1}\right) \ldots a\left(p_{r}\right) d p_{1} \ldots d p_{r},
$$

where $f$ is a function in $\mathscr{F}^{r}$. By estimates of the form (2.8) we have that $A_{i}\left(H+b_{r}\right)^{-r / 2}$ is bounded. Moreover by Lemma 2.1 $\left(A_{i}\right)_{t}\left(H+b_{r}\right)^{-r / 2} \Omega$ converges strongly to the limits $\left(A_{i}\right)_{ \pm}\left(H+b_{r}\right)^{-r / 2} \Omega$, as $t \rightarrow \pm \infty$. On the other hand $\left(A_{i}\right)_{t}\left(H+b_{r}\right)^{-r / 2} \Omega$ is uniformly norm bounded in $t$. Since the mapping $f \rightarrow\left(A_{i}\right)_{t}\left(H+b_{r}\right)^{-r / 2}$ is norm continuous, because of estimates of the type (2.8), from $f$ in $\mathscr{F}^{r}$ into the set of all bounded operators on $\mathcal{F}$, and moreover uniformly norm bounded in $t$, we can approximate strongly the vectors $\left(A_{i}\right)_{t}\left(H+b_{r}\right)^{-r / 2} \Omega$ by vectors which are Iinear combinations of vectors of the form $\left(a^{\#}\left(h_{1}\right) \ldots a^{\#}\left(h_{r}\right)\right) t^{\Omega}$, and this approximation is uniform in $t$.

Since we have proven above that

$$
\begin{aligned}
& \left(a^{\frac{\|}{*}}\left(h_{1}\right) \ldots a^{\#}\left(h_{r}\right)\right) t \Omega\left(a^{\frac{H}{\#}}\left(h_{1}\right) \ldots a^{\#}\left(h_{r}\right)\right)_{ \pm} \Omega= \\
= & \mathbb{W}_{ \pm} a^{\#}\left(h_{1}\right) \ldots a^{\frac{1}{\hbar}}\left(h_{r}\right) \Omega_{0} \text { as } t \rightarrow \pm \infty,
\end{aligned}
$$

an $\frac{\epsilon}{3}$ argument and the fact that the $W_{ \pm}$are bounded operators complete the proof of the lemma.

We now define the scattering amplitude for $n$ incoming particles with momentum distributions $g_{1}, \ldots, g_{n}$ in $\mathscr{F}^{1}$ and $m$ outgoing particles with momentum distributions $h_{1}, \ldots, h_{m}$ in $F^{1}$, as

$$
S_{n, m}\left(g_{1} \ldots g_{n} ; h_{1} \ldots h_{m}\right)=\left(a_{+}^{*}\left(g_{1}\right) \ldots a_{+}^{*}\left(g_{n}\right) \Omega, a_{-}^{*}\left(h_{1}\right) \ldots a_{-}^{*}\left(h_{m}\right) \Omega\right),
$$
which, by $(2.6)$, is equal to

$$
\left(a *\left(g_{1}\right) \ldots a^{*}\left(g_{n}\right) \Omega_{0}, W_{+}^{* W} a^{*}\left(h_{1} \ldots a^{*}\left(h_{m}\right) \Omega_{0}\right) .\right.
$$

Therefore the scattering matrix is given by the scattering operator

$$
S=W_{+}^{*} W_{-}
$$


defined on the whole Fock space $\mathscr{F}$.

$S$ maps $\mathscr{F}$ into $\mathcal{F}$, and is a contraction: $\|S\| \leq 1$. Moreover

$S$ commutes with $\mathrm{H}_{\mathrm{O}}:\left[\mathrm{S}, \mathrm{H}_{\mathrm{O}}\right]=0$.

3. The asymptotic series for the asymptotic fields and the scattering operator.

Since all the integrals we shall consider will always be understood as strong ones, we shall mostly omit to write this specification in the considerations of this section.

Let $A$ be any operator of the form (2.7).

By Lemma 2.4 we have, for any $y \in D_{p}(j+1)+\frac{k}{2}$ :

$$
A_{ \pm} \Psi=A \Psi-i \lambda \int_{0}^{ \pm \infty} e^{-i s H}\left[V, e^{i s H}{ }_{0} A e^{-i s H} 0\right] e^{i s H_{\Psi}} d s
$$

Hence

$$
A_{+} \Psi=A_{-} \Psi-i \lambda \int_{-\infty}^{\infty} e^{-i s H_{1}}\left[\mathrm{~V}, e^{i s H_{0}} \mathrm{Ae}^{-i \mathrm{sH}} 0\right] e^{i s \mathrm{sH}_{\Psi} \mathrm{ds}} .
$$

We have also, from Lemma 2.2:

$$
A_{t} \Psi=A \Psi-i \lambda \int_{0}^{t} e^{-i s H}\left[V, e^{i s H_{0}} e^{-i s e_{0}}\right] e^{i s H_{\Psi d s}} \text {. }
$$

Introducing therefore on the right hand side of this equality the expression obtained from (3.1) for $A_{\Psi}$ in terms of $A_{-} \Psi$, we obtain:

$$
\begin{aligned}
& A_{t} \Psi=A_{-} \Psi+i \lambda \int_{0}^{-\infty} e^{-i s H}\left[V, e^{i s H_{0}} A e^{-i s H_{0}}\right] e^{i s H} \Psi d s \\
& -i \lambda \int_{0}^{t} e^{i s H}\left[\mathrm{~V}, e^{i s{ }} \mathrm{Ae} e^{-i s H_{0}}\right] e^{i s H} \psi d s= \\
& =A_{-} \Psi-i \lambda \int_{-\infty}^{t} e^{-i s H}\left[V, e^{i s H_{0}} A e^{-i s H_{0}} \rho e^{i s H_{\Psi}} \Psi s\right. \text {. }
\end{aligned}
$$




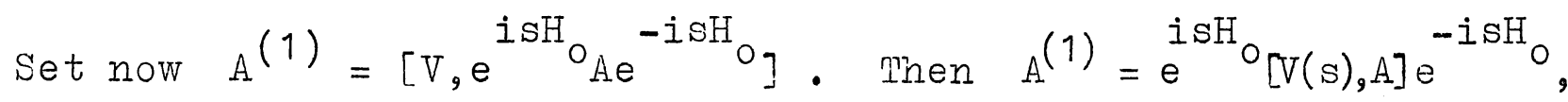
hence $A^{(1)}$ is of the form (2.7). Hence by Lemma 2.4 applied to $A^{(1)}$ we obtain:

$$
A_{-}^{(1)} x=A^{(1)} x-i \lambda \int_{0}^{-\infty} e^{-i \sigma H}\left[V, e^{i \sigma H_{O}}(1) e^{-i \sigma H} o\right] e^{i \sigma H_{1}} x d s,
$$

for any $x \in D$

$$
p(j+1)+\frac{k}{2} \cdot
$$

Choose now $\Psi$ to be any element in the dense subset $D_{p(j+1)+\frac{k}{2}}$ of $D_{p j+\frac{k}{2}}$. Then $e^{i t H_{\psi}}$ belongs also to $D_{p(j+1)+\frac{k}{2}}$ and introducing $\quad \chi=e^{i t H_{\Psi}}, A^{(1)} x$ as given by (3.5) into the last integral in (3.4), we get:

$$
\begin{aligned}
& A_{t} \Psi=A_{-} \Psi-i \lambda \int_{-\infty}^{t} e^{-i s H_{A}(1)} e^{i s H_{\Psi}} d s=A_{-} \Psi-i \lambda \int_{-\infty}^{t} e^{-i s H_{A}(1)} e_{-}^{i s H_{\Psi}} d s- \\
& -(i \lambda)^{2} \int_{-\infty}^{t} d s e^{-i s H} \int_{0}^{-\infty} d \sigma e^{-i \sigma H_{1}}\left[V_{g} e^{i \sigma H_{A}} o_{A}(1) e^{-i \sigma H_{0}}\right] e^{i \sigma H_{2} i s H_{\Psi}} .
\end{aligned}
$$

Hence, since all integrals are strongly convergent,

$$
\begin{aligned}
& A_{+} \Psi=A_{-}-i \lambda \int_{-\infty}^{+\infty} e^{-i s H_{A}(1)} e^{i s H_{\Psi}} d s= \\
& +(-i \lambda)^{2} \int_{-\infty}^{\infty} d s e^{-i s H} \int_{-\infty}^{0} d \sigma e^{-i \sigma H_{1}}\left[V_{,} e^{i \sigma H_{O_{A}}(1)} e^{-i \sigma H_{0}}\right] e^{i \sigma H_{e}} e^{i s H_{\Psi}} .
\end{aligned}
$$

Changing now the integration variables in the last integral, we get:

$$
\begin{aligned}
& A_{+} \Psi=A_{-} \Psi-i \lambda \int_{-\infty}^{\infty} d s e^{-i s H_{A}}(1) e^{i s H_{\psi}}+ \\
& +(-i \lambda)^{2} \int_{-\infty}^{\infty} d s \int_{-\infty}^{s} d s_{1} e^{-i s_{1} H_{1}}\left[V_{g} e^{i s_{1} H_{O_{A}}(1)} e^{-i s_{1} H_{0}}\right] e^{i s_{1} H_{\psi}} .
\end{aligned}
$$

We remark that $A^{(2)} \equiv\left[V, e^{i s_{1} H_{0}}(1) e^{-i s_{1} H_{O}}\right]$ is equal to $e^{i s_{1} H_{O}}\left[V\left(s_{1}\right), A^{(1)}\right] e^{-i s_{1} H_{o}}$, and hence is again of the form (2.7). 
Therefore we can derive (3.5) with $A^{(2)}$ instead of $A^{(1)}$, for any $x \in D_{p(j+2)+\frac{k}{2}}$. Hence if the original $\Psi$ is chosen to be in $D_{p(j+2)+\frac{k}{2}}$, then (3.5) with $x=e^{i s_{1} H_{\psi}}$ and $A^{(2)}$ instead of $A^{(1)}$ permits to replace the third term on the right hand side of (3.7) by the sum of two terms. Proceeding in this way, for $\psi \in D_{p(j+n)+\frac{k}{2}}$ we obtain the similar formulae, involving $A^{(I)}$, where $A^{(I)}$ are defined recursively by

$$
A^{(I)}=\left[V, e^{i s_{I-1} H_{A}}(I-1) e^{-i s_{I-1}{ }^{H}}\right] \text { for } I=1,2, \ldots, n \text {. }
$$

By lemma 2.4 one has

$$
e^{-i s H} A_{-}^{(I)} e^{i s H} x=\left(e^{-i s O_{A}(I)} e^{i s H_{0}}\right)_{-} x, \text { for any } x \in D_{p(j+I)+\frac{k}{2}}{ }^{\circ}
$$

These relations are used to rewrite the expressions involving $A^{(I)}$ according to the following example:

$$
\left.\int_{-\infty}^{\infty} d s e^{-i s H_{A}(1)} e^{i s H_{\Psi}}=\int_{-\infty}^{\infty} d s\left(e^{-i s H_{A}}(1) e^{i s H_{0}}\right)\right)_{-} \Psi=\int_{-\infty}^{\infty} d s\left[V(s), A_{-} \Psi .\right.
$$

We formulate now the

\section{Theorem 3.1}

Let $A$ be any operator of the form (2.7) (in particular any operator of the form $a^{\frac{\| 4}{\| t}}(h)$ or $a^{\frac{\pi}{t^{*}}}\left(h_{1}\right) \ldots a^{\frac{*}{\#}}\left(h_{k}\right)$, with $\left.h_{i} \in \mathscr{F}^{1}, i=1 \ldots k\right)$.

Then $A_{t}=e^{-i t H} e^{i t H} O_{A} e^{-i t H} e^{i t H}$ converges strongly on $D_{p(\mathbb{N}+1+j)+\frac{k}{2}}$ to $A_{ \pm}$as $t \rightarrow \pm \infty, \mathbb{N}=1,2, \ldots$.

The limits for $t \rightarrow+\infty$ and those for $t \rightarrow-\infty$ are related to each other by the following asymptotic expansions:

$$
\begin{gathered}
\left.A_{+} \Psi=A_{-} \Psi+\sum_{I=1}^{\mathbb{N}}(-i \lambda)^{I} \int_{t_{1} \leq \ldots \leq t_{1}} \ldots \int\left\{\left[V\left(t_{1}\right), \ldots v\left(t_{y}\right), A\right] \ldots\right]\right\}-\Psi d t_{1} \ldots d t_{1}+ \\
+R_{N+1}\left(A_{+}\right) \Psi,
\end{gathered}
$$


for any $\Psi \in D_{p(N+1+j)+\frac{k}{2}}$, where $\left\{\left[V\left(t_{I}\right), \ldots\left[V\left(t_{1}\right), A\right] \ldots\right]\right\}-\Psi=$ $\left.=\underset{t \rightarrow-\infty}{s-\lim _{t \rightarrow \infty}} e^{-i t H_{1}} e^{i t H} 0\left[V\left(t_{I}\right), \ldots,\left[V\left(t_{1}\right), A\right] \ldots\right]\right\} e^{-i t H_{0}} e^{i t H_{\Psi}}$ and

$$
\begin{aligned}
& \mathrm{R}_{\mathbb{N}+1}\left(\mathrm{~A}_{+}\right) \Psi=(-i \lambda)^{\mathbb{N}+1} \int_{\sigma \leq t_{\mathbb{N}} \leq \ldots \leq t_{1}} e^{-i \sigma \mathrm{H}_{1}} e^{i \sigma \mathrm{H}} \mathrm{O}\left[\mathrm{V}(\sigma),\left[V\left(t_{\mathbb{N}}\right), \ldots\left[V\left(t_{1}\right), A\right] \ldots\right.\right. \\
& \ldots] \mathrm{e}^{-i \sigma \mathrm{H}_{0}} \mathrm{e}^{i \sigma \mathrm{H}_{\Psi} d \sigma d t_{1} \ldots d t_{\mathbb{N}}} .
\end{aligned}
$$

All integrals are strongly convergent. The remainder $R_{N+1}\left(A_{+}\right) \Psi$ satisfies the estimate

$$
\left\|R_{N+1}\left(A_{+}\right) \Psi\right\| \leq|\lambda|^{N+1} C_{N+1}\left\|\left(H+b_{2 p}(N+1+j)+k\right){ }^{p(N+1+j)+\frac{k}{2}} \Psi\right\|,
$$

with $\mathrm{C}_{\mathrm{N}+1}$ independent of $\lambda$ and $\Psi$.

Noreover the operators $A_{t}$ and their limits on $D_{p(N+1+j)+\frac{k}{2}}$ a.s $t \rightarrow+\infty$ are expressed in terms of the time zero quantities A by the asymptotic series:

$$
A_{t} \Psi=A \Psi+\sum_{I=1}^{N}(-i \lambda)^{I} \int_{0 \leq t_{N} \leq \ldots \leq t_{1} \leq t}\left[V\left(t_{\mathbb{N}}\right), \ldots\left[V\left(t_{1}\right), A\right] \ldots\right] \Psi d t_{1} \ldots d t_{I}+R_{N+1}^{0}\left(A_{t}\right) \Psi,
$$

for any $t<\infty$ and also for $t=+\infty$, with $A_{t=\infty} \equiv A_{+}$, and for any $\Psi \in D$

$$
p(N+1+j)+\frac{k}{2}
$$

The remainder $R_{\mathrm{N}+1}^{q}\left(A_{t}\right)$ is given by

$$
\begin{aligned}
& R_{\mathbb{N}-1}^{i}\left(A_{t}\right)=(-i \lambda)^{N+1} \int_{0}^{t} d s \int_{0}^{s} d s_{1} \ldots \int_{0}^{s_{I N-1}} d s_{N} e^{-i s_{N} H} e^{i s_{N} H_{0}} \\
& \left\{e^{-i s_{N-1} H_{0}}\left[V\left(s_{N}-s_{N-1}\right),\left[V\left(s_{N-1}\right), \ldots[V(s), A] \ldots\right] e^{i s_{N}-1 H_{0}}\right\}\right. \\
& e^{-i s_{N} H_{0}} e^{i s_{N} H_{\Psi}},
\end{aligned}
$$

and estimated by

$$
\left\|R_{\mathbb{N}+1}^{\prime}\left(A_{t}\right) \Psi\right\| \leq|\lambda|^{N+1} C_{N+1}^{d}\left\|\left(H+b_{2 p}(N+1+j)+k\right)^{p(N+1+j)+\frac{k}{2}} \psi\right\|,
$$

with $\mathrm{C}_{\mathbb{N}+1}^{p}$ independent of $\lambda$ and $\psi$. 
Similar formulae hold for $t \rightarrow-\infty$ instead of $t \rightarrow+\infty$.

Remark: For the case $A=a^{\#}(h)$ these expansions are of the form of the so called Dyson-Schwinger ones. Each term is given here in terms of known quantities, namely the time zero fields and the interaction in the interaction picture. Hence these expansions provide a way to compute, in the sense of asymptotic series, the asymptotic fields (and e.g. polynomials in them).

Proof: (3.8) has been proven already before the statement of the Theorem. Note that the expression for \{\}$_{-} \Psi$ as strong limit holds because of Lemma 2.4. The estimate on the remainder (3.9) follows from the fact that $\hat{A}=\left[V(\sigma),\left[V\left(t_{\mathbb{N}}\right), \ldots\left[V\left(t_{1}\right), A\right] \ldots\right]\right]$ satisfies $\left\|\hat{A}(\underline{\mathbb{N}}+1)^{-\left(p(\mathbb{N}+1+j)+\frac{k}{2}\right)}\right\| \leq C_{\mathbb{M}}(1+|t|)^{-\mathbb{M}}$, as a consequence of the higher order estimates (2.4) (as seen similarly as in the proof of Lemma 2.3) and $\left\|(\underline{N}+1)^{-\left(p(N+1+j)+\frac{k}{2}\right)} e^{-i \sigma H_{0}} e^{i \sigma H_{\Psi}}\right\| \leq$ const. $\left\|\left(H+b_{2 p}(N+1+j)+k\right)^{p(N+1+j)+\frac{k}{2}} \Psi\right\|$, by the same estimates. The asymptotic expansion (3.11) is established in a similar way as (3.8), starting form (3.3) and inserting in this relation the expression for $A^{(1)}=\left[V, e^{i s H_{A}} e^{-i s H_{0}} 0\right]$ given by $A_{-S}^{(1)} x=A^{(1)} \chi-i \lambda \int_{0}^{S} e^{-i \sigma H}\left[V, e^{i \sigma H_{0}} o_{A}(1) e^{-i \sigma H} 0\right] e^{i \sigma H} x d \sigma$, for any $x \in D_{p(\mathbb{N}+1+j)+\frac{\pi}{2}}$

Consider now the scattering operator $S=W_{-}{ }^{*} W_{+}$. Since it is bounded, it is determined by its values on a dense set of states. By linearity it is sufficient to compute $S B \Omega_{0}$, where $B=a^{*}\left(h_{1}\right)$.. $\ldots a^{*}\left(h_{m}\right)$, with $h_{i} \in \mathscr{F}^{-1}, i=1, \ldots, m$, and $m$ arbitrary. We 
incluade the case $B \Omega_{0}=\Omega_{0}$ by the convention that $B$ stands for the identity for $m=0$.

We have

$$
S B \Omega_{0}=W_{-}^{*} W_{+} B \Omega_{0}
$$

and since, by Lemma 2.4,

$$
W_{+} B \Omega_{0}=B_{+} \Omega
$$

we get

$$
\text { S } B \Omega_{0}=W_{-}^{*} B_{+} \Omega \text {. }
$$

On the other hand $\mathrm{B}_{+} \Omega$ is given, according to Theorem 3.1, by:

$$
\begin{aligned}
& B_{+} \Omega=\sum_{j=0}^{n}(-i \lambda)^{j} \int_{t_{j} \leq \ldots \leq t_{1}}\left\{\left[v\left(t_{j}\right),\left[\ldots\left[V\left(t_{1}\right), B\right] \ldots\right]\right\}-\Omega d t_{1} \ldots d t_{j}+\right. \\
& \sigma \leq t_{n} \leq \ldots \leq t_{1} \\
& {\left[V(\sigma),\left[V\left(t_{n}\right),\left[\ldots\left[V\left(t_{1}\right), B\right] \ldots\right] e^{-i \sigma H_{0}} e^{i \sigma H} \Omega d \sigma d t_{1} \ldots d t_{n},\right.\right.}
\end{aligned}
$$

where all the integrals are strongly convergent. By Lemma 2.5 we have:

$$
\begin{aligned}
& \left\{\left[V\left(t_{j}\right),\left[\ldots,\left[V\left(t_{1}\right), B\right], \ldots\right]\right\}-\Omega=\right. \\
& =V_{-}\left\{\left[V\left(t_{j}\right),\left[\ldots,\left[V\left(t_{1}\right), B\right] \ldots\right]\right\} \Omega_{0} .\right.
\end{aligned}
$$

Inserting this into (3.11) we get

$$
\begin{aligned}
B_{+} \Omega=\sum_{j=0}^{n}(-i \lambda)^{j} \int_{t_{j} \leq \ldots . \leq t_{j}}\left\{\left[V\left(t_{j}\right),\left[\ldots\left[V\left(t_{1}\right), B\right] \ldots\right]\right\} \Omega_{0} d t_{1} \ldots d t_{j}+\right. \\
\\
+R_{n+1}\left(B_{+}\right) \Omega,
\end{aligned}
$$

$$
\begin{gathered}
S B \Omega_{0}=\sum_{j=0}^{n}(-i \lambda)^{j} \int_{t_{j} \leq \ldots \leq t_{1}} W_{-}^{*} W-\left\{\left[V\left(t_{j}\right),\left[\ldots\left[V\left(t_{1}\right), B\right] \ldots\right]\right\} \Omega_{0} d t_{1} \ldots d t_{j}\right. \\
+W R_{n+1}\left(B_{+}\right) \Omega .
\end{gathered}
$$

But $W_{-}^{*} W_{-}=1$, since $W_{-}$is an isometry. 
Hence

$$
\begin{array}{r}
S B \Omega_{0}=\sum_{j=0}^{n}(-i \lambda)^{j} \int_{t}\left\{\left[V\left(t_{j}\right),\left[\ldots\left[V\left(t_{1}\right), B\right] \ldots\right] \Omega_{0} d t_{1} \ldots d t_{j}+\right.\right. \\
+\ldots t_{1}+R_{n+1}(S B) \Omega,
\end{array}
$$

where $R_{n+1}(S B) \Omega=W_{-}^{*} R_{n+1}\left(B_{+}\right) \Omega$ and thus, by (3.9):

$$
\begin{gathered}
R_{n+1}(S B) \Omega=W_{-}^{*}(-i \lambda)^{n+1} \int_{\sigma \leq t_{n} \leq \ldots \leq t_{1}} e^{-i \sigma H_{1}} e^{i \sigma H_{0}} \\
{\left[V(\sigma),\left[V\left(t_{n}\right), \ldots,\left[V\left(t_{1}\right), B\right] \ldots\right] e^{-i \sigma H_{0}} e^{i \sigma H_{1}} d \sigma d t_{1} \ldots d t_{n} .\right.}
\end{gathered}
$$

We have the

\section{Theorem 3.2}

The scattering operator $S$, defined by (2.13), has an asymptotic power series expansion in $\lambda$ when applied to any vector $\psi$ of the dense set of vectors of the Fock space, obtained by applying the polynomial algebra of to the Fock vacuum $\Omega_{0}$. The expansion is given, for $\Psi=a^{*}\left(h_{1}\right) \ldots a^{*}\left(h_{m}\right) \Omega_{0}$, by

$$
\begin{aligned}
& S a *\left(h_{1}\right) \ldots a^{*}\left(h_{m}\right) \Omega_{0}=\sum_{j=0}^{\mathbb{N}}(-i \lambda)^{j} \int_{j \leq \ldots \leq t_{1}} \\
& \left\{\left[V\left(t_{i}\right), \ldots\left[V\left(t_{1}\right), a *\left(h_{1}\right) \ldots a *\left(h_{m}\right)\right] \ldots\right]\right\} \Omega_{0} a t_{1} \ldots a t_{j} \\
& \quad+R_{N+1}\left(S a *\left(h_{1}\right) \ldots a *\left(h_{m}\right)\right) \Omega,
\end{aligned}
$$

where $R_{N+1}\left(S a *\left(h_{1}\right) \ldots a *\left(h_{m}\right)\right) \Omega=(-i \lambda)^{N+1} W_{-} \int e^{-i \sigma H_{0}} e^{i \sigma H_{0}}$

$$
\sigma \leq t_{N} \leq \ldots \leq t_{1}
$$

$$
\left[V(\sigma),\left[V\left(t_{N}\right), \ldots,\left[V\left(t_{1}\right), a^{*}\left(h_{1}\right) \ldots a^{*}\left(h_{m}\right)\right] \ldots\right]\right] e^{-i \sigma H_{O}} e^{i \sigma H_{2}} \Omega d \sigma d t_{1} \ldots d t_{N^{*}}
$$

All integrals are strongly convergent.

There wists an $\epsilon>0$ such that for all $0 \leq \lambda \leq \epsilon$ the remainder $R_{I N+1}\left(S a *\left(h_{1}\right) \ldots a^{*}\left(h_{m}\right)\right) \Omega$ satisfies the estimate: 


$$
\left\|R_{N+1}\left(S a^{*}\left(h_{1}\right) \ldots a^{*}\left(h_{m}\right)\right) \Omega\right\| \leq \lambda^{N+1} C_{N+1}
$$

where $\mathrm{C}_{\mathrm{N}+1}^{\prime}$ is a constant independent of $\lambda$.

Proof: The expansion coincides with $(3.15),(3.16)$, with $B=$ $=a^{*}\left(h_{1}\right) \ldots a^{*}\left(h_{m}\right), N=n, R_{N+1}(S B) \Omega=R_{N+1}\left(S a^{*}\left(h_{1}\right) \ldots a^{*}\left(h_{m}\right)\right) \Omega$, and hence has been proven already. The estimate on the remainder is obtained as follows. $W_{-}^{*}$ is partial isometric, hence, by (3.16) and (3.10):

$$
\left\|R_{N+1}\left(S a^{*}\left(h_{1}\right) \ldots a^{*}\left(h_{m}\right) \Omega\right)\right\| \leq|\lambda|^{N+1} C_{N+1}\left\|\left(H+b_{2 p(N+1)+k}\right)^{p(N+1)+\frac{k}{2}} \Omega\right\|_{9}(3.20)
$$

where $C_{N+1}$ is independent of $\lambda$.

But $H \Omega=E \Omega$, and the lowest eigenvalue $E$ is known to be bounded uniformly in $\lambda$ for $\lambda$ in a finite interval $[0, \epsilon]$.

Moreover $b_{2 p}(N+1)+k$ is also bounded for $\lambda \in[0, \varepsilon]$ (see $[14]$ ). Hence $\left\|\left(H+b_{2 p}(N+1)+k\right)^{p(N+1)+\frac{k}{2}} \Omega\right\| \leq$ const., where the constant is independent of $\lambda$, for $\lambda \in[0, \epsilon]$. This then, inserted into (3.20), proves (3.19) and the Theorem.

From Theorem 3.2 we have immediately the asymptotic expansion of the S-matrix :

\section{Theorem 3.3}

The S-matrix is determined by matrix elements of the form

$$
\begin{aligned}
& S_{n_{,} m}\left(g_{1} \ldots g_{n} ; h_{1} \ldots h_{m}\right)=\left(a_{-}^{*}\left(g_{1}\right) \ldots a_{-}^{*}\left(g_{n}\right) \Omega,\right. \\
& \left.a_{+}^{*}\left(h_{1}\right) \ldots a_{+}^{*}\left(h_{m}\right) \Omega\right)=\left(a^{*}\left(g_{1}\right) \ldots a^{*}\left(g_{n}\right) \Omega_{0},\right. \\
& S_{*}^{*}\left(h_{1} \ldots a^{*}\left(h_{m}\right) \Omega_{0}\right),
\end{aligned}
$$

9) It is even known [14] that the Rayleigh-Schrødinger series for Fis is asymptotic power series in $\lambda$, uniquely Borel summable to its sum $E$. Also the asymptotic expansion for $\Omega$ is known [14]. This could also be inserted for $\Omega$ in the expression (3.18) of $R_{N+1}\left(S a *\left(h_{1}\right) \ldots a^{*}\left(h_{m}\right)\right) \Omega$. 
where $g_{i} \in \mathscr{F}^{1}, h_{j} \in \mathscr{F}^{1}, i=1, \ldots, n ; j=1, \ldots, m$.

These matrix elements have asymptotic power series expansions in $\lambda$ given by:

$$
\begin{aligned}
& S_{n, m}\left(g_{1} \ldots g_{n} ; h_{1} \ldots h_{m}\right)=\sum_{I=0}^{N}(-i \lambda)^{I} \int_{t_{I} \leq \ldots \leq t_{1}}\left(a^{*}\left(g_{1}\right) \ldots a^{*}\left(g_{n}\right) \Omega_{0},\right. \\
& {\left[V\left(t_{1}\right), \ldots\left[V\left(t_{1}\right), a^{*}\left(h_{1}\right) \ldots a^{*}\left(h_{m}\right)\right] \ldots\right] \Omega_{0} d t_{1} \ldots a t_{I}+R_{N+1}\left(S_{n_{1}, m}\right)}
\end{aligned}
$$

where $R_{\mathbb{N}+1}\left(S_{n, m}\right)=\left(a^{*}\left(g_{1}\right) \ldots a^{*}\left(g_{n}\right) \Omega_{0}, R_{N+1}\left(S a^{*}\left(h_{1}\right) \ldots a^{*}\left(h_{m}\right) \Omega\right)\right)=$

$$
\begin{aligned}
= & (-i \lambda)^{\mathbb{N}+1}\left(a^{*}\left(g_{1}\right) \ldots a^{*}\left(g_{n}\right) \Omega_{0}, W^{*}-\int_{\sigma \leq t_{N} \leq \ldots \leq t_{1}} e^{-i \sigma H_{e}} e^{i \sigma H} O V(\sigma),\left\ulcorner V\left(t_{N}\right), \ldots\right.\right. \\
& {\left.\left.\left[V\left(t_{1}\right), a^{*}\left(h_{1}\right) \ldots a^{*}\left(h_{m}\right)\right] \ldots\right]\right] e^{-i \sigma H_{0}} e^{i \sigma H_{1}} \Omega d \sigma d t_{1} \ldots d t_{N} . }
\end{aligned}
$$

The remainder satisfies the estimate

$$
\left|\mathrm{P}_{\mathrm{N}+1}\left(\mathrm{~S}_{\mathrm{n}, \mathrm{m}}\right)\right| \leq|\lambda|^{\mathrm{N}+1} \mathrm{C}_{\mathrm{N}+1}
$$

with $\mathrm{C}_{\mathbb{N}+1}^{\prime \prime}$ independent of $\lambda$.

Remark 1: The terms, up to the arbitrary order $\mathbb{N}$, in the asymptotic expansion of $S_{n, m}$ given in Theorem 3.3 are expressed in terms of the Fock vacuum, the free time zero fields and the interaction $\mathrm{V}$ in the interaction picture, and can thus be computed. One checks easily that the asymptotic expansion for $\mathrm{S}_{\mathrm{n}, \mathrm{m}}$ is different from the one of a constant, since the terms of order larger or equal 1 do not vanish identically. Since to a given function there is only one asymptotic expansion, this proves that (as to be expected!) space cut-off polynomial interactions in two spacetime dimensions have non trivial scattering.

10) The analoguous result was proven in [1b] for Nelson's type models and in [11c] for non polynomial interactions. 
Remark 2: In order to prove that the series given by the right hand side of (3.21) for $N \rightarrow \infty$ is actually uniquely summable to the sum $S_{n, m}$, additional information would be needed, e.g. in the form of strong enough estimates on the remainder (with respect to the order $\mathbb{N}$ ) and analyticity of $S_{n, m}$ in $\lambda$ in some suitable complex sector. 11) We have proven the summability of the series (3.21) for $N \rightarrow \infty$ to $S_{n, m}$, in the strong form of $S_{n, m}$ being analytic for $\lambda$ in a disk, for non polynomial interactions with space and ultraviolet cut-off, in all space-time dimensions, in $\operatorname{Ref.}[11 \mathrm{c}]$.

4. Unitarity of the scattering operator in the sense of asymptotic series.

We shall now construct the asymptotic power series expansions for $S^{*} S$ and $S^{*}$, using the asymptotic power series expansion of $S$ given by Theorem 3.2. Consider first $S^{*} S=W_{+}^{*} W_{-}$. Since $W_{-}$is bounded, we can form $W_{-} S a^{*}\left(h_{1}\right) \ldots a^{*}\left(h_{m}\right) \Omega$, which, by Theorem 3.2 , is given by:

$$
W_{-} S a^{*}\left(h_{1}\right) \ldots a^{*}\left(h_{m}\right) \Omega=\sum_{j=0}^{N}(-i \lambda)^{j} \int_{t_{j} \leq \ldots \leq t_{1}} W_{-} A \Omega_{0} d t_{1} \ldots d t_{j}+W R_{N+1} \Omega_{9} \quad \text { (4.1) }
$$

where we have set $A \equiv\left[V\left(t_{j}\right), \ldots\left[V\left(t_{1}\right), a^{*}\left(h_{1}\right) \ldots a^{*}\left(h_{m}\right)\right] \ldots\right]$,

11) Results of this type have been obtained by B. Simon and I. Rosen-B. Simon [14] for other quantities in these models, including the vacuum energy and the equal time Wightman functions. 
and where we have also interchanged the integrations and the multiplication by $W_{\text {- }}$, which is allowed, since all integrals are strongly convergent and $W_{-}$is bounded.

By Lemma 2.5 we have

$$
W_{-} A \Omega_{0}=A_{-} \Omega \text {, }
$$

where $A_{-}=\underset{t \rightarrow-\infty}{s-l i m} e^{-i t H_{e}} e^{i t H} \mathrm{Ae}^{-i t \mathrm{O}_{\mathrm{H}}} \mathrm{e}^{i t H}$.

A has the form of the operators covered by Theorem 3.1 and $\Omega$ belongs to the set of vectors considered in the same theorem, since $\Omega$ belongs to the domain of all powers of $H$.

Hence we have:

$$
\begin{array}{r}
A_{-} \Omega=A_{+} \Omega+\sum_{k=1}^{M}(i \lambda)^{k} \int_{\tau_{k} \geq \cdots \geq \tau_{1}}\left\{\left[V\left(\tau_{k}\right), \ldots\left[V\left(\tau_{1}\right), A\right] \ldots\right]\right\}+\Omega \\
d \tau_{1} \ldots d \tau_{K}+R_{M+1}\left(A_{-}\right) \Omega,
\end{array}
$$

with

$$
\begin{aligned}
& R_{M+1}\left(A_{-}\right) \Omega=(i \lambda)^{M+1} \int e^{-i \tau H_{e}} e^{i \tau H_{0}}\left[V(\tau),\left[V\left(\tau_{\mathbb{M}}\right) \ldots[\right.\right. \\
& \tau \geq \tau_{\mathbb{N}} \geq \cdots \geq \tau_{1} \\
& \left.\left.\left.V\left(\tau_{1}\right), A\right] \ldots .\right]\right] e^{-i \tau H_{0}} e^{i \tau H_{\Omega}} d \tau d \tau \ldots d \tau_{M I} \text {, }
\end{aligned}
$$

where all the integrals are strongly convergent. Foom Lemma 2.5 we have:

$$
\left\{\left[V\left(\tau_{k}\right), \ldots,\left[V\left(\tau_{1}\right), A\right] \ldots\right]\right\}_{+} \Omega=W_{+}\left\{\left[V\left(\tau_{k}\right), \ldots\left[V\left(\tau_{1}\right), A\right] \ldots\right]\right\} \Omega_{0} \cdot
$$

Introducing this into (4.3) we obtain

$$
\begin{aligned}
& W A \Omega_{0}=A_{-} \Omega=A_{+} \Omega+\sum_{k=1}^{\mathbb{M}}(i \lambda)^{k} \int_{\tau_{k} \geq \ldots \geq \tau_{1}} W_{+}\left\{\left[V\left(\tau_{k}\right), \ldots,\left[V\left(\tau_{1}\right), A\right] \ldots\right] \Omega_{0}\right. \\
& +R_{M+1}\left(A_{-}\right) \Omega .
\end{aligned}
$$

Insert now this expression for $W_{-} A \Omega_{0}$ into the sum on the right hand side of $(4.1)$. We obtain: 


$$
\begin{aligned}
& W_{-} S a *\left(h_{1}\right) \ldots a *\left(h_{m}\right) \Omega= \\
& =\sum_{j=0}^{\mathbb{N}}(-i \lambda)^{j} \int_{t \leq \ldots \leq t_{1}}\left(\sum_{j=0}^{\mathbb{N}}(i \lambda)^{k} \int_{\tau_{k} \geq \ldots \geq \tau_{1}} W_{+}\left\{\left[V\left(\tau_{k}\right), \ldots\left[V\left(\tau_{1}\right), A\right] \ldots\right]\right\} \Omega_{0} d \tau_{1} \ldots d \tau_{k}\right. \\
& +\sum_{j=0}^{\mathbb{N}}(-i \lambda)^{j} \int_{t_{j} \leq \ldots \leq t_{1}} R_{\mathbb{N}+1}\left(A_{-}\right) \Omega d t_{1} \ldots d t_{j}+\mathbb{W} R_{N+1} \Omega
\end{aligned}
$$

where all the integrals are again strongly convergent. We apply now the bounded operator $W_{+}^{*}$ to both sides of $(4.6)$. Because of the strong convergence of the integrals and the boundedness of $W_{+}^{*}$ we can bring $W_{+}^{*}$ under the integrals. Since, on the other hand, $W_{+}^{*} W_{-}=S^{*}$, we obtain:

$$
\begin{aligned}
& S^{*} S^{*}\left(h_{1}\right) \ldots a^{*}\left(h_{m}\right) \Omega=W_{+}^{*} S^{*}\left(h_{1}\right) \ldots a^{*}\left(h_{m}\right) \Omega= \\
& =\sum_{j=0}^{\mathbb{N}} \sum_{k=0}^{\mathbb{N}}(-i \lambda)^{j}(i \lambda)^{k} \int_{j} \quad \int\left\{\left[V\left(\tau_{k}\right)_{, \ldots . .}\left[V\left(\tau_{1}\right), A\right] \ldots\right]\right\} \Omega_{0} \\
& t_{j} \leq \ldots \leq t_{1} \tau_{k} \geq \ldots \geq \tau_{1} \quad d t_{1} \ldots d t_{j} d \tau_{1} \ldots d \tau_{k} \\
& +\sum_{j=0}^{N}(-i \lambda)^{j} \int_{t \leq \ldots \leq t_{1}} W_{+}^{*} R_{\mathbb{M}+1}\left(A_{-}\right) \Omega d t_{1} \ldots d t_{j}+S * R_{N N+1} \Omega
\end{aligned}
$$

where again all integrals are strongly convergent. Introduce now the following sequence of characteristic functions for all $k=0,1,2, \ldots$ :

$$
\begin{aligned}
& \quad x_{0}=1 \text { and } x_{k}\left(t_{1}, \ldots, t_{k}\right)=1 \text {, if } \\
& t_{k} \leq t_{k-1} \leq \ldots \leq t_{1}
\end{aligned}
$$

$x_{k}\left(t_{1}, \ldots, t_{k}\right)=0$ otherwise.

Then one has the formula $([15])$ :

$$
0=\sum_{k=0}^{n}(-1)^{k} x_{n-k}\left(t_{n}, \ldots, t_{k+1}\right) x_{k}\left(t_{1}, \ldots, t_{k}\right),
$$

valid for any $n=0,1,2, \ldots$ and all $t_{1}, \ldots, t_{n}$, except for the case where some of the arguments coincide. This formula is easily proven by induction. 
We can on the other hand write $(4.7)$ as follows:

$$
\begin{aligned}
& S^{*} S a^{*}\left(h_{1}\right) \ldots a^{*}\left(h_{m}\right) \Omega_{0}=\sum_{k=0}^{N}(-i \lambda)^{K} \sum_{j=0}^{N}(i \lambda)^{j} \\
& \int \ldots \int x_{j}\left(t_{k+j} \cdots, t_{k+1}\right) x_{k}\left(t_{1} \ldots t_{k}\right)\left[V\left(t_{k+j}\right), \ldots\left[V\left(t_{k+1}\right),\right.\right. \\
& {\left[V\left(t_{k}\right), \ldots\left[V\left(t_{1}\right), a^{*}\left(h_{1}\right) \ldots a^{*}\left(h_{m}\right)\right] \ldots\right] \Omega_{0} d t_{1} \ldots d t_{k+j}+R,}
\end{aligned}
$$

with

$$
R=\sum_{j=0}^{\mathbb{N}}(-i \lambda)^{j} \int_{\substack{j \leq \ldots \leq t_{1} \\ t_{j} \leq W^{*} R_{M+1}}}\left(A_{-}\right) \cap d t_{1} \ldots d t_{j}+S^{*} R_{N+1} \Omega .
$$

Hence

$$
\begin{aligned}
& S^{*} S a^{*}\left(h_{1}\right) \ldots a^{*}\left(h_{m}\right) \Omega_{0}=\sum_{n=0}^{N+\mathbb{M}}(i \lambda)^{n}\left[\ldots \int_{k=0}^{n}(-1)^{k}\right. \\
& x_{n-k}\left(t_{n}, \ldots, t_{k+1}\right) x_{k}\left(t_{1}, \ldots, t_{k}\right)\left[V\left(t_{n}\right), \ldots\left[V\left(t_{1}\right), a *\left(h_{1}\right) \ldots a *\left(h_{m}\right)\right] \ldots\right] \Omega_{0} d t_{1} \ldots d t_{n}+R .
\end{aligned}
$$

Using now the identity (4.8), we see that the integrands are zero for $0<\mathrm{n} \leq \mathbb{N}+\mathbb{M}$.

Therefore we have

$$
S * S a *\left(h_{1}\right) \ldots a *\left(h_{m}\right) \Omega_{0}=a *\left(h_{1}\right) \ldots a *\left(h_{m}\right) \Omega_{0}+R,
$$

where $R$ is given by $(4.10)$.

For $R$ we can easily find an estimate, using the facts that $\mathrm{W}_{+}^{*}$ is a partial isometry, $S^{*}$ is a contraction, $R_{\mathbb{M}+1}\left(A_{-}\right) \Omega$ is given by (4.3) and $R_{N+1} \Omega$ is estimated by (3.19).

We have thus, for $|\lambda| \leq \epsilon$ :

$$
\begin{aligned}
& \|R\| \leq|\lambda|^{\mathbb{M}+1}(\mathbb{N}+1) \max _{j=0_{g \circ 0, \mathbb{N}}} \int_{t_{j} \leq \ldots \leq t_{1}} \int_{\tau \geq \tau_{\mathbb{M}} \geq \ldots \geq \tau} \|\left[V(\tau),\left[V\left(\tau_{\mathbb{M}}\right), \ldots\right.\right. \\
& \ldots\left[V\left(\tau_{1}\right),\left[V\left(t_{j}\right) \ldots\left[V\left(t_{1}\right), a^{*}\left(h_{1}\right) \ldots a^{*}\left(h_{m}\right)\right]\right] e^{-i \tau H_{0}} e^{i \tau I H} \Omega \| d t_{y} \ldots d t_{j} d \tau d \tau_{1} \ldots d \tau_{\mathbb{M}} \cdot\right.
\end{aligned}
$$

But the operator under the norm is of the form (2.7) of those estimated in Lemma 2.3. Hence the norm is bounded by $C(\mathbb{M}, j, m, r)(1+|\tau|)^{-r}$, for any $r$, and thus the integrals on 
the norm are convergent.

We have therefore proven the estimate

$$
\|\mathrm{R}\| \leq|\lambda|^{\mathrm{M}+1} \mathrm{~K}_{\mathbb{M}}
$$

where $\mathrm{K}_{\mathrm{M}}$ is independent of $\lambda$.

The orem 4.1

Let $D$ be the dense set of vectors in Fock space obtained by applying on the Fock vacuum $\Omega_{0}$ the polynomial algebra $\rho$ consisting of all polynomials in the creation and annihilation operators $a^{\frac{\hbar}{1}}\left(h_{1}\right) \ldots a^{\frac{\hbar}{\#}}\left(h_{k}\right)$, with arbitrary square integrable $h_{i}$, $i=1, \ldots, k$, ( $k$ being any arbitrary integer). On $D$ the operators $S^{*} S$ and $S^{*}$ are asymptotic, in the strong topology, to the identity operator, for small values of $\lambda$. S is the scattering operator defined by (2.13).

Thus, for any $\psi \in D$ :

$$
\text { and } \begin{aligned}
S^{*} S \Psi & =\Psi+R \\
S^{*} \Psi & =\Psi+R^{\prime},
\end{aligned}
$$

with remainders $R, R^{\prime}$ which satisfy

$$
\|R\| \leq|\lambda|^{N_{K_{N}}}, \quad\left\|R^{*}\right\| \leq|\lambda|^{N_{K_{N}}^{q}} \text {. }
$$

for any $N=0,1,2, \ldots$, where $K_{N}, K_{N}^{8}$ are independent of $\lambda$, $\lambda$ real.

Hence the scattering operator and the S-matrix are "asymptotic unitary" in the sense that $S * S$ and $S S^{*}$ are given, on the dense domain $D$, by asymptotic power series in the coupling constant which are asymptotic, in the strong topology, to the identity.

Equivalently: For any vector $\Psi \in D$, the vectors $S^{*} S \Psi$ and 
SS*4 are strongly differentiable in $\lambda$, to all orders, with all derivatives at $\lambda=0$ equal to zero.

Proof: The asymptotic series for $S * S$ has been derived above, as well as the estimate for the remainder $R$.

The analogous result for $S^{*}$ is obtained by completely parallel arguments, since the passage from $S$ to $S^{*}$ is obtained by interchanging $W_{+}$with $W_{-}$, and our asymptotic series, like the ones in Theorem 3.1, are derived for all cases.

Remark: $K_{N}$ and $K_{N}^{1}$ might depend on the vector $\Psi$ and on the order $N$. To prove complete unitarity, and not only unitarity in the sense of asymptotic series, one would need e.g. stronger estimates for $\lambda$ complex, on the various remainders on which our estimate for $R$ is based.

The asymptotic series for $S^{*} S$ and $S^{*}$ yield immediately the usual unitarity relations for S-matrix elements between any vectors in the above dense set $D$ of states. Here we have not only "unitarity in every order" but also an estimate on the remainders of the relevant expansions in the coupling constant, since these are proven to be asymptotic series.

If we analyze the proofs of all the asymptotic expansions of this paper, we see that ingredients needed are the essential selfadjointness of the Hamiltonian $\mathrm{H}$ on $\mathrm{D}\left(\mathrm{H}_{0}\right) \cap \mathrm{D}(\mathrm{V})$, the spatial cut-off in $V$ and a strong control on $V$ of the type of the one provided by Rosen's estimates (2.4). The same proofs work in the same way for the classes of space and ultraviolet cut-off models, in any space-time dimensions, considered in [10], i.e. for inter- 
action densities of the form $\mathrm{P}_{\mathrm{b}}+\mathrm{P}_{\mathrm{y}}+\mathrm{P}_{\mathrm{w}}$, where $\mathrm{P}_{\mathrm{b}}, \mathrm{P}_{\mathrm{y}}, \mathrm{P}_{\mathrm{w}}$ are polynomials in finitely many boson fields resp. boson and fermion resp. fermion fields (even degrees in the fermion fields and linearity in the boson fields for $\mathrm{P}_{\mathrm{y}}$ ). In the same way all the proofs work for the Nelson's type models discussed in [1]. Finally they can also be carried through for the class of non-polynomial interactions of the form $V=\int_{|\vec{x}| \leq I} e^{i s \varphi_{\varepsilon}(\vec{x})} d \nu(s) d \vec{x}$, with an ultraviolet cut-off boson field $\varphi_{\varepsilon}$ and where the symmetric measure $\nu(s)$ is finite with bounded support and satisfies $\int|s| d|\nu|(s)<\infty$. This is a subclass of the interactions studied (also in the infinite volume $(1 \rightarrow \infty)$, limit) in [11] (where also references to previous work are given). For these models the analyticity of the S-matrix elements $S_{n, m}$ in $\lambda$, for small $\lambda$, has also been proven in [11c]. The asymptotic series is thus in this case convergent to the analytic functions $S_{n, m}$. In this case it is moreover proven [11c] to coincide with the linked cluster expansion of the S-matrix. The present paper yields, for these nonpolynomial models, the additional information of the unitarity of the scattering operator (and the S-matrix) in the sense of asymptotic series, for small values of the coupling constant $\lambda$.

\section{Acknowledgements}

The first named author (S.A.) would like to use the opportunity to thank the Institute of Mathematics of Oslo University for the warm hospitality and for providing an excellent working atmosphere. 


\section{References}

[1]a) R. Høegh-Krohn, Asymptotic fields in some models of quantum field theory, III, Journ.Math.Phys.11, 185-188 (1970).

b) S. Albeverio, Scattering theory for some models of quantum fields I, Princeton University Preprint 1971 (to appear in Journ.Mathem.Physics); II, Helv.Phys.Acta (Fierz Festschrift) 45, 303-321 (1972).

[2]a) B. Simon, Quantum mechanics for Hamiltonians defined as quadratic forms, Princeton University Press, Princeton (1971).

b) K. Hepp, Quantum scattering theory of multiparticle systems, lectures given at the Conference "Mathematics of Contemporary Physics", Bedford College, London (to appear).

[3] S. Albeverio, on bound states in the continuum of N-body systems and the virial theorem, Annals of Phys., 11, 167$276(1972)$.

[4] R.J. Iorio - M.O' Carroll, Asymptotic completeness for multiparticle Schrödinger Hamiltionians with weak potentials, Commun.math. Phys. 27, 137-145 (1972).

[5] R. Lavine, Completeness of the wave operators in the repulsive N-body problem, Preprint, Institute for Advanced Study, Princeton, 1972.

[6] R. Høegh-Krohn, Partly gentle perturbation with application to perturbation by annihilation-creation operators, Proc.Nat.Ac.Sci. 58, 2187-2192 (1967).

R. Høegh-Krohn, Partly gentle perturbations with application to perturbations by annihilation-creation operators, Commun. on Pure and Appl.Math., 21, 313-142 (1968).

R. Høegh-Krohn, Gentle perturbations by annihilation-creation operators, Commun. on Pure and Appl.Math. 21, 343-357 (1968). 
[7] J. Glimm - A. Jaffe, Quantum field theory models, in: Statistical Mechanics and Quantum Field Theory, les Houches Summer School 1970, Ed. C. de Witt, R. Stora, Gordon and Breach, New York, 1972.

[8] R. Høegh-Krohn, On the spectrum of the space cut-off :P( $\varphi)$ : Hamiltonian in two space-time dimensions, Commun.math. Phys. 21, 256-260 (1971).

[9] Y. Kato - N. Mugibayashi, Asymptotic fields in model field theories I, Progr. Theor.Phys. 45, 628-639 (1971).

[10] R. Høegh-Krohn, On the scattering operator for quantum fields, Commun.Math.Phys. 18, 109-126 (1970).

[11]a) R. Høegh-Krohn, Boson fields under a general class of cut-off interactions, Commun. Math.Phys. 12, 216-225 (1969).

b) S. Albeverio-R. Høegh-Krohn, Uniqueness of the physical vacuum and the Wightman functions in the infinite volume limit for some non polynomial interactions, Preprint Series, Mathematics Institute, Oslo, Aug.1972 (to appear in Commun. Math. Phys.).

c) S. Albeverio-R. Høegh-Krohn, The scattering matrix for some non-polynomial interactions I, Preprint Series, Mathmatics Institute, OsIo, Oct.1972.

[12]a) I. Rosen, $A \lambda \varphi^{2 n}$ field theory without cut-offs Commun. Math. Phys. 16, 157-183 (1970).

b) I. Rosen, The $\left(\varphi^{2 n}\right)_{2}$ quantum field theory: higher order estimates, Comm. Pure Appl. Math. 24, 417-157 (1977).

[13] K. Hepp, Theorie de la renormalisation, Lecture Notes in Physics, Ed. by J. Ehlers, K. Hepp, H.A. Weidenmuller, Springer-Verlag, Berlin, 1969. Ch.1.

[14]a) B. Simon, Borel summability of the ground-state energy in spatially cut-off $\left(\varphi^{4}\right)_{2}$, Phys.Rev.Iett. 25, 1583-1586 (1970). 
b) I. Rosen - B. Simon, The $\left(\varphi^{2 n}\right)_{2}$ field Hamiltonian for complex coupling constant, Trans.Am.Math.Soc. 165, 365-379 (1972).

[15] K. Hepp, Renormalization Theory, in: Statistical Mechanics and Quantum Field Theory, Les Houches Summer School 1970, Ed. C. De Witt, R. Stora, Gordon and Breach, New York, 1971. 